\title{
Studies on the preparation of multi-monomer grafted PP by one-step extrusion and the blends with PVC
}

\author{
L. L. Hou ${ }^{*}$, M. Zhao ${ }^{2}$ \\ ${ }^{1}$ Hydrochem (S) PTE LTD, A Hyflux Group Company, 339339, Singapore \\ 2Department of Fire Technology, the Chinese People's Armed Police Academy, Hebei 065000, P.R. China
}

Received 1 September 2007; accepted in revised form 22 October 2007

\begin{abstract}
Firstly, a novel grafted polypropylene (PP) was prepared by one step free-radical melt grafting method in a single-screw extruder. It was shown that the addition of St to the melt-grafting system as a comonomer could significantly enhance the grafting degree of MMA onto PP and reduced the degradation of PP matrix by means of FTIR and MFR tests, respectively. Then, the extruded multi-monomer grafted PP, as a component, was directly blended with poly(vinyl chloride) (PVC), denoted as gPP/PVC. The corresponding improved compatibility was examined. Due to the addition of gPP the tensile strengths of gPP/PVC blends increased significantly in comparison to pure polypropylene/poly(vinyl chloride) blends (PP/PVC), while the impact strengths remained unchanged. The DSC results also suggested that the compatibility of $\mathrm{PP} / \mathrm{PVC}$ blends were improved largely.
\end{abstract}

Keywords: polymer blends and alloys, multi-monomer grafted, polypropylene, poly(vinyl chloride), compatibility

\section{Introduction}

Graft copolymers of PP have been widely used as compatibilizers in blends of PP with other plastics such as polyamides and polyesters, which were generally produced by the melt free radical grafting of monomers onto the PP chains. Various monomers have been used for melt grafting reaction. The most commonly employed monomers were maleic anhydride, glycidyl methacrylate and vinyl and acrylic monomers containing reactive functional groups [1-3].

However, the grafting yields of those monomers were usually low; and the PP matrix would undergo $\beta$-scission induced PP chain degradation in course of the grafting of polar monomers [4-6]. To obtain high grafting yields and to reduce side reactions, it was essential that the macroradicals react with the grafting monomer before they undergo side reactions. Recently, a so-called 'comonomer concept' was developed to improve the grafting yields of glycidyl methacrylate (GMA) onto PP and PE (polyethylene) [6,8]. The idea of using a comonomer was associated with the commonly accepted fact that a free radical process starts with the formation of macroradicals along the polymer chains by a so-called hydrogen abstraction mechanism. These macroradicals might subsequently follow two competing pathways. They could either initiate the grafting of the monomer or undergo chain scission. The latter depends strongly on the nature of the polymer backbone. In the case of PP, the main side reaction was $\beta$-scission associated with PP macroradicals [6], which caused a reduction in the molecular weight of the polymer.

Styrene (St) was found to be a good comonomer to promote the melt free radical grafting and reduce the chain scission of PP matrix. It was believed to relate to the high reactivity towards PP macroradi- 
cals and the stability of the resulting product. St reacted rapidly with $\mathrm{PP}$ macroradicals and the resulting styryl macroradicals copolymerized readily with grafting monomer $[4,6,9]$.

In this work we provide a detailed investigation on melt free radical grafting of methylmethacrylate (MMA) onto PP with St as a comonomer in a single-screw extruder and study its ability to improve the compatibility of otherwise incompatible blends. Specifically, the multi-monomer grafted polymer was synthesized, and then the grafted PP was directly blended with PVC to study the improved compatibility between PP and PVC. The mechanical proprieties, thermal behavior and morphology of the blends were investigated in detail. Meantime, the pure PP/PVC blend was prepared for comparison.

\section{Experimental}

\subsection{Materials}

$\mathrm{PP}$ and PVC used in this study were commercial products, the former was supplied by Daqing Petrochemical Factory (T30S, Heilongjiang, China), with a melt flow rate (MFR) of $3.5 \mathrm{~g} / 10 \mathrm{~min}$; the latter was the product of Baoding Electrochemical Plant (SG-6, suspension type, $K$ value $=65-63$, Hebei, China). The corresponding processing agents of PVC: tribasic lead sulfate (TLS), dibasic lead phosphate (DLP), stearic acid (SA), paraffin wax (PW) (industrial grade). The grafting monomers were MMA (Xintong Fine Chemical Co., Ltd, Tianjin, China) and St (Fuxing Chemical Factory, Beijing, China). Dicumyl peroxide (DCP) was chosen as an initiator purchased from Shanghai chemical reagent company (Shanghai, China).

\subsection{Preparation and purification of the multi-monomer grafted PP}

The radical initiator, MMA and St were introduced in a beaker firstly. The resultant mixture was dryblended with PP pellets before charged into the extruder at room temperature for about 30 minutes so that they could be absorbed by the PP matrix. Then the reactions of melt grafting St and/or MMA onto PP were carried out in a single screw extruder of type XJ-20 (Scientific Research Instrument Factory, Jilin University, China). The screw diameter
$(D)$ was $20 \mathrm{~mm}$ and the screw diameter-to-extruder length ratio excluding the die was 20 . The extruding temperature was set at the range from 160 to $210^{\circ} \mathrm{C}$.

The crude grafted samples were first dissolved in hot toluene and then precipitated in excess acetone at room temperature, the grafted PP and pure PP were precipitated whereas the homo- and co-polymer, such as poly (methylmethacrylate) (PMMA), polystyrene (PSt) and poly(styrene-co-methyl methacrylate) (PSt-co-PMMA) remained soluble. After that, the precipitated samples, denoted as PP-g-(St-co-MMA), were filtered and dried under vacuum at $80^{\circ} \mathrm{C}$ for $12 \mathrm{~h}$.

\subsection{Characterization of modified PP}

Fourier Transform Infrared Spectroscopy (FTIR) was carried out with a FTS-40 spectrometer (BIORAD, Co., USA) in the range between 4000 and $500 \mathrm{~cm}^{-1}$, with a resolution of $2 \mathrm{~cm}^{-1}$ at room temperature, which was used to determine the grafting yields of MMA and/or St onto PP.

MFR measurements of the purified PP samples were carried out using $\mu$ PXRZ-400C Melt Indexer (Jilin University Scientific Research Instrument Factory, Jilin, China) at $230^{\circ} \mathrm{C}$ with a load of $2.16 \mathrm{~kg}$ weight according to the ASTM D1238 standard.

\subsection{Measurements of mechanical properties of blends}

PVC with its processing agents at a fixed weight ratio (PVC:TLS:DLP:SA:PW = 100:2:1:0.5:0.5) were first dry-mixed by high-speed mixer, followed by melt blending in a two roll mixing mill for plastics (XKR-160, Zhanjiang Machine Company, Guangdong, China) to plasticate for $4 \mathrm{~min}$, and the plastication of PP or grafted PP (gPP) had the same procedure as that of PVC. PVC was added to plasticated PP or gPP followed by blending for another $4 \mathrm{~min}$. After that, the blends were compressed into sheets with a hydraulic press at $180^{\circ} \mathrm{C}$ and $10 \mathrm{MPa}$ for $5 \mathrm{~min}$, which is designated as $\mathrm{PP} / \mathrm{PVC}$ or gPP/PVC, respectively. The corresponding sheets were directly milled into standard testing specimens according to ASTM D638 and ASTM D 256 standard. Prior to each processing step, all sheets 
were dried in a vacuum oven at $80^{\circ} \mathrm{C}$ for $12 \mathrm{~h}$. Notched Izod impact strength was measured by a XCJ-40 impact tester (Chengde Laboratory Instrument Works, Hebei, China). Tensile testing was performed on an LJ-500N tensile test machine (Chengde Laboratory Instrument Works, Hebei, China) at a speed of $10 \mathrm{~mm} / \mathrm{min}$.

\subsection{DSC investigation}

Differential scanning calorimeter (DSC) measurements were carried out on a DT-40 Modulated DSC (Shimadzu, Kyoto, Japan) calibrated by indium standards. All the measurements were performed from room temperature to $200^{\circ} \mathrm{C}$ at a heating/cooling rate of $20^{\circ} \mathrm{C} / \mathrm{min}$ under nitrogen atmosphere, and maintained at that temperature for $5 \mathrm{~min}$ to eliminate any previous thermal history. The glass transition temperature $\left(T_{g}\right)$ was determined in the second heating scan.

\section{Results and discussion}

\subsection{FTIR analysis of PP-g-(St-co-MMA)}

The FTIR spectra of the pure PP, PP-g-MMA and PP-g-(St-co-MMA) are shown in Figure 1. The characteristic absorption peaks at $1730 \mathrm{~cm}^{-1}$ corresponded to the absorption of the carbonyl groups of

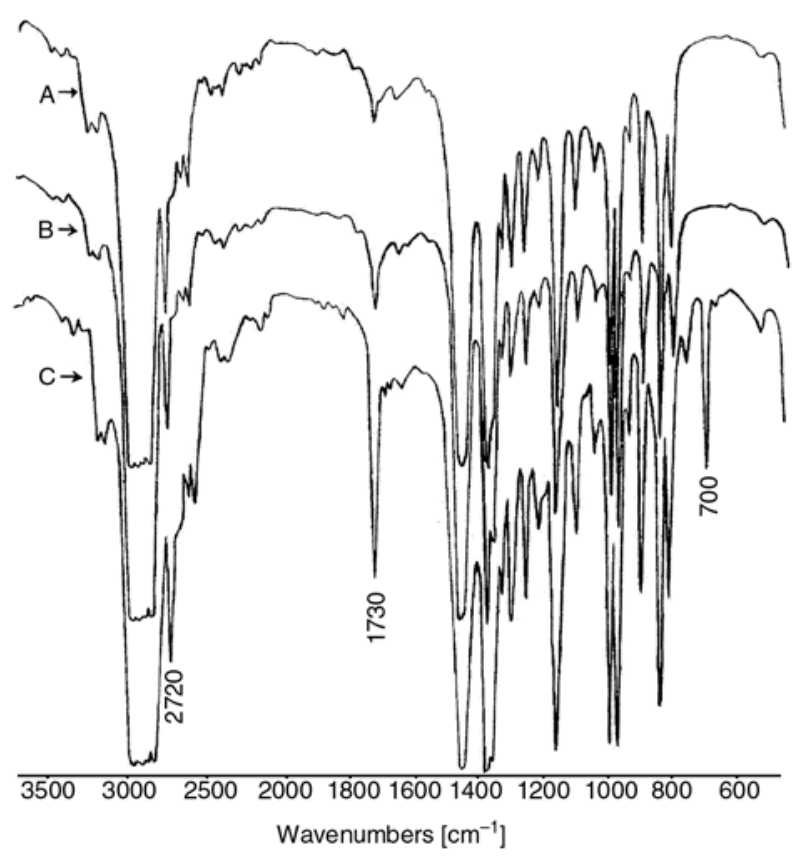

Figure 1. FT-IR Spectra of PP, PP-g-MMA and PP-g-(St-co-MMA). A: pure PP, B: PP-MMA, C: PP-g-(St-co-MMA)
MMA. The peaks at 700 and $2720 \mathrm{~cm}^{-1}$ are attributed to the characteristic absorption of the St and PP skeleton, respectively. The above-mentioned results illustrated that both MMA and St were grafted onto PP.

To obtain the quantitative results, the FTIR absorbance ratio technique is applied to determine the grafting yields $[4,12,13]$. The absorption peak at $2720 \mathrm{~cm}^{-1}$ was chosen as an internal reference and that at $1730 \mathrm{~cm}^{-1}$ were used to determine the grafting degree of MMA. The peak at $700 \mathrm{~cm}^{-1}$ corresponding to the stretching of the hydrogen atoms of the mono-substituted aromatic ring of $\mathrm{St}$ was chosen for measuring the amount of grafted St. The relative absorbance ratio (RAR) values of the areas of the peaks at 1730 (or 700) and $2720 \mathrm{~cm}^{-1}$ showed the relative grafting degree of MMA (or $\mathrm{St})$.

\subsection{Preparation and characterization of PP-g-(St-co-MMA)}

The important contribution of using $\mathrm{St}$ as comonomer to enhance the MMA grafting yields and reduce $\beta$-scission induced PP chain degradation is illustrated in Figures 2 and 3 below.

Figures 2 and 3 show the RAR (relative absorbance ratio) and MFR values as a function of the molar ratio of $[\mathrm{St}] /[\mathrm{MMA}]$ for a given concentration of MMA charged (6.0 phr), respectively. As seen from Figure 2, in the absence of St, the RAR value, which was an indicator of the grafting degree of MMA, was very low due to the low reactivity of MMA. Adding St as comonomer, the RAR values

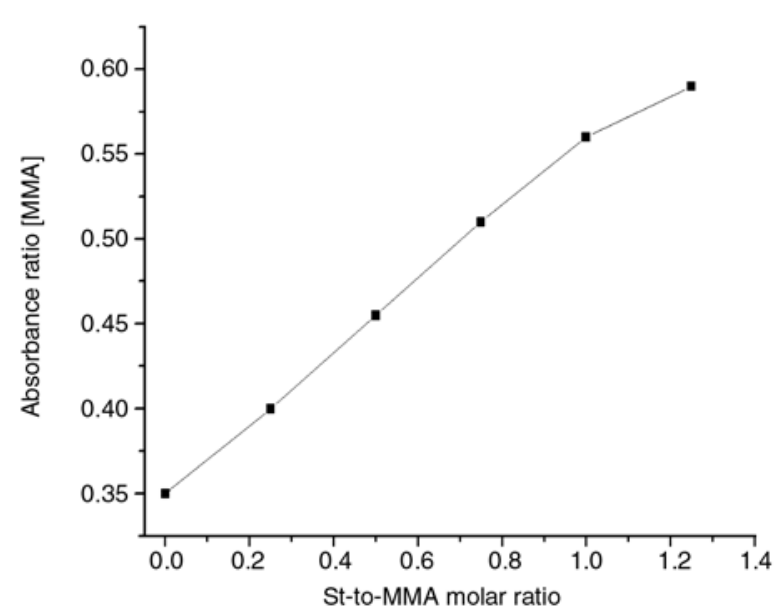

Figure 2. Effect of monomer molar ratios on grafting degree of the grafted PP 


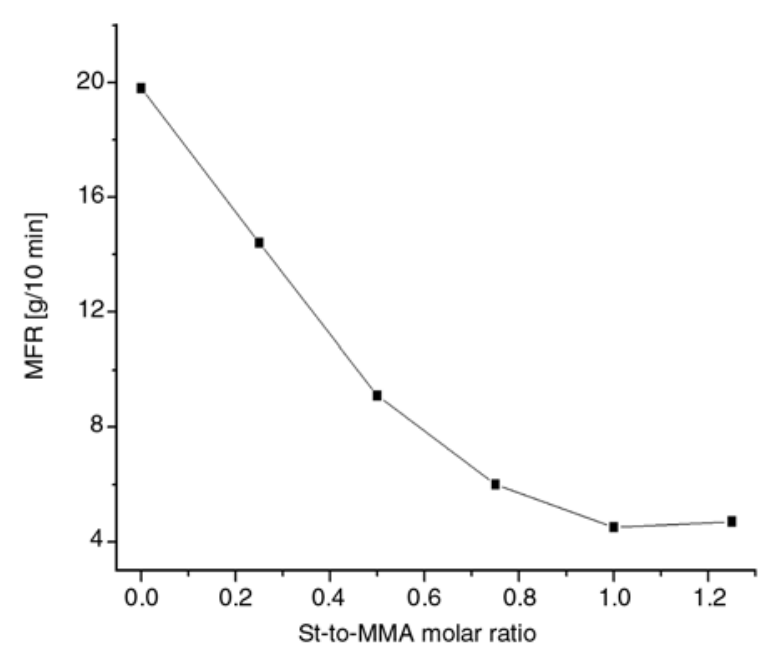

Figure 3. Effect of monomer molar ratios on MFR value of the grafted PP

ascended linearly with increasing the molar ratio of the two monomers until the ratio reached $1: 1$; thereafter the change slowed down. This might be caused by the more and more homo- and co-polymer [6], such as PMMA, PSt and PSt-PMMA, all these byproducts were obtained simultaneously with the grafting reaction. When the molar ratio was small, the grafting reaction was dominant; all above side reactions were negligible. With the molar ratio increasing, especially beyond $1: 1$, these side reactions affected the grafting yields declining to a large degree. The RAR values of MMA, on the other hand, always had larger value than that of St. It was interesting phenomenon to notice for us, because of the higher reactivity of St than that of MMA. In Figure 3, the MFR value decreased drastically from 19.8 to 4.6 as the molar ratio increased $1: 1$ and then leveled off.

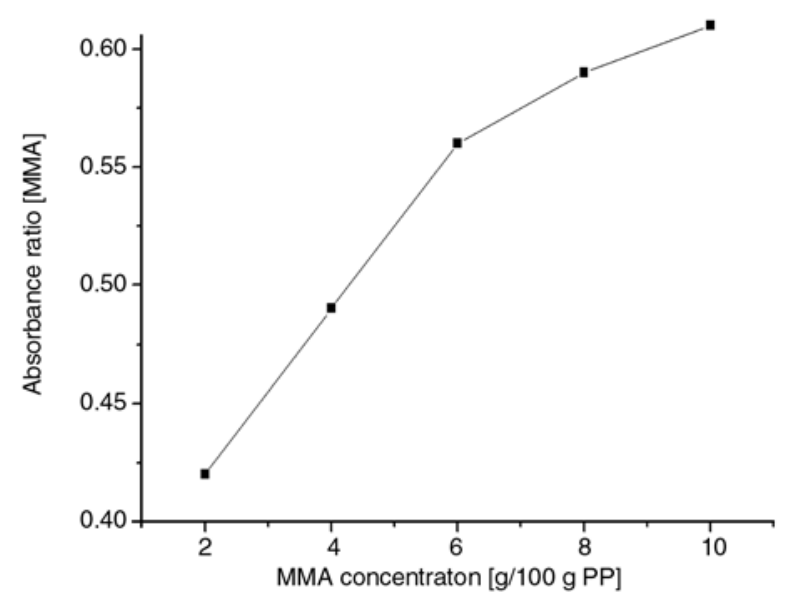

Figure 4. Effect of MMA concentration on grafting degree of the grafted PP

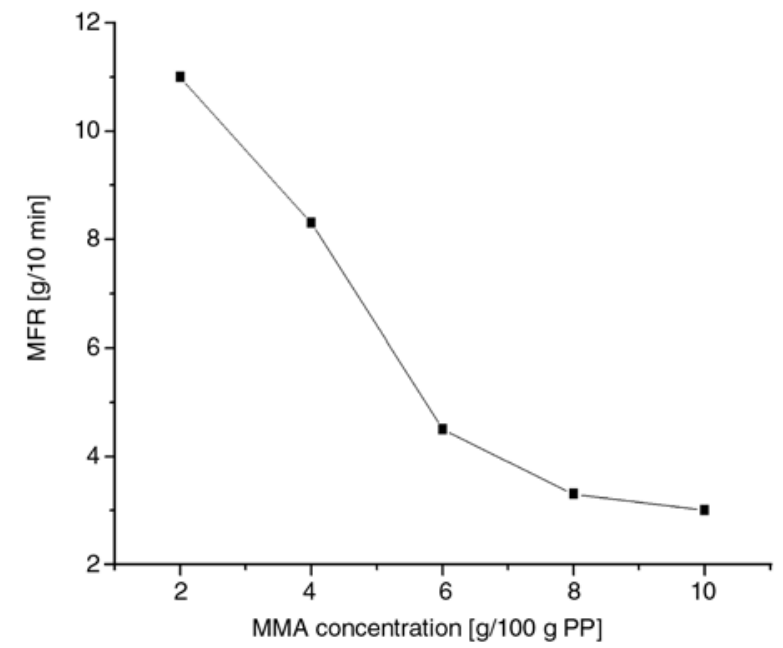

Figure 5. Effect of MMA concentration on MFR value of the grafted PP

For a given condition, DCP concentration and the molar ratio of $[\mathrm{St}] /[\mathrm{MMA}]$ were fixed at $0.6 \mathrm{phr}$ and 1:1, respectively. Figure 4 shows that the more the monomers (MMA or St) were added, the higher the RAR values of MMA and St. Figure 5 shows the effect of grafting monomer concentration on MFR values of the grafted PP obtained at the same conditions as Figure 4. The MFR values of the grafted PP decreased with increasing monomer concentration, and some had similar values to that of pure PP, which meant that the addition of $\mathrm{St}$ reduced the degradation of PP effectively.

From Figures 3 and 5, it was clear to see that the degradation of PP chain was reduced significantly with the St content adding; otherwise, the ability for St to promote the MMA grafting yields might be obtained from Figure 4. In another word, the comonomer, St, could reduce $\beta$-scission of PP to a great extent and provided an additional freedom to control different monomers' grafting yields in some systems.

In order to investigate the influences of DCP on grafting degree and MFR values of the grafted PP, the monomer molar ratio was fixed at 1:1 and the two grafting monomers content were $6 \mathrm{phr}$ based on PP. It could be seen from Figure 6 that the changes of the RAR values were large. In the beginning, because the most DCP was consumed by the grafting reaction, the RAR value increased significantly and reached a peak value at $0.6 \mathrm{phr}$ DCP. However, Figure 7 shows that the MFR values had a little change when the content of DCP was ranged from 0.2 to $0.8 \mathrm{phr}$. With the content of 


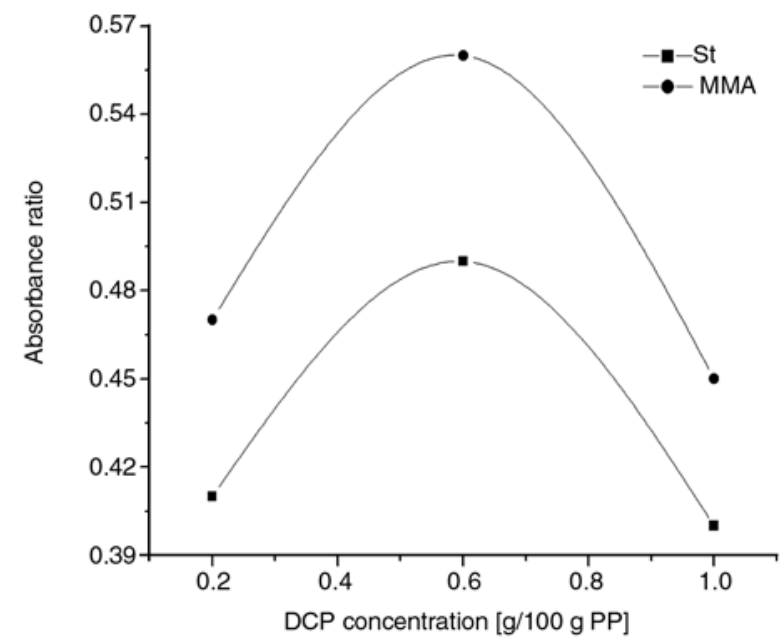

Figure 6. Effect of DCP on grafting degree of the grafted PP

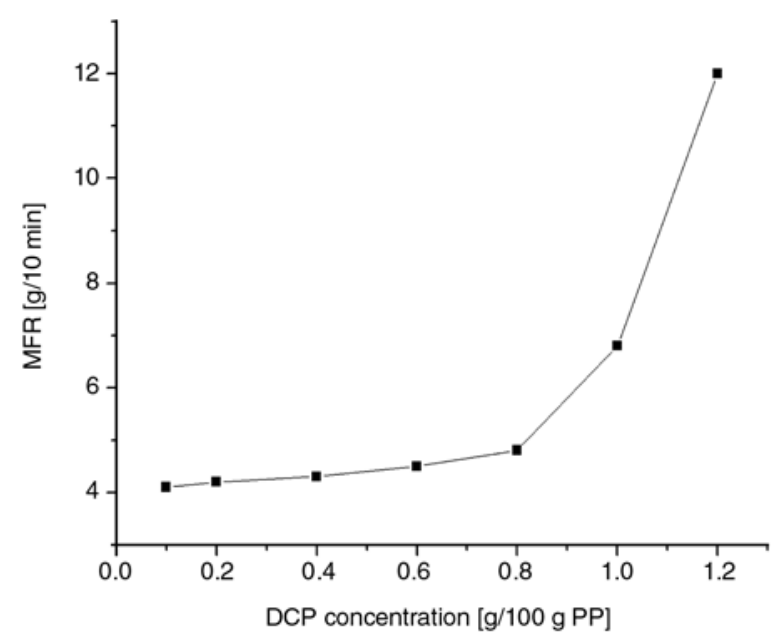

Figure 7. Effect of DCP on MFR value of the grafted PP

DCP increased consecutively, the RAR values decreased rapidly and the MFR values increased; the more the concentration of DCP, the more the decrease of the RAR values and the increase of the MFR values. This might be related to the increased degradation of the PP matrix. Therefore, the surplus of the DCP content was harmful to the graft reactivity. Thus, the optimal concentrations for St, MMA and DCP is 6.0, 6.0, and 0.6, respectively based on PP, the corresponding grafted PP are directly blended with PVC to study their compatibility. Additionally, it was interesting to note that, the RAR values of MMA were higher than that of St and no longer limited by a plateau but increased with increasing initial concentration of MMA. The similar phenomenon was found at other grafting systems. [6-9]. As a result, a comonomer St could
Table 1. Mechanical properties of pure PP, PVC, gPP and their blends

\begin{tabular}{|r|r|r|c|c|}
\hline $\begin{array}{r}\text { PP } \\
{[\mathbf{g}]}\end{array}$ & $\begin{array}{c}\text { PVC } \\
{[\mathbf{g}]}\end{array}$ & $\begin{array}{c}\text { gPP } \\
{[\mathbf{g}]}\end{array}$ & $\begin{array}{c}\text { Tensile strength } \\
{[\mathbf{M P a}]}\end{array}$ & $\begin{array}{c}\text { Impact strength } \\
{\left[\mathbf{K J} / \mathbf{m}^{2}\right]}\end{array}$ \\
\hline 0 & 100 & 0 & 45.00 & - \\
\hline 100 & 0 & 0 & 35.17 & 2.014 \\
\hline 0 & 0 & 100 & 34.72 & 1.777 \\
\hline 20 & 80 & 0 & 9.16 & 1.599 \\
\hline 40 & 60 & 0 & 9.39 & 1.447 \\
\hline 60 & 40 & 0 & 22.74 & 1.483 \\
\hline 80 & 20 & 0 & 28.06 & 1.746 \\
\hline 0 & 80 & 20 & 19.99 & 1.630 \\
\hline 0 & 60 & 40 & 7.57 & 1.659 \\
\hline 0 & 40 & 60 & 23.61 & 1.684 \\
\hline 0 & 20 & 80 & 34.31 & 1.794 \\
\hline
\end{tabular}

provide an additional freedom to control different monomers' grafting yields in some systems.

\subsection{Mechanical proprieties of PP/PVC and gPP/PVC blends}

PP and PVC are two kinds of important plastics, they were widely used in many fields. Due to their different polarity, poor adhesion, and high interfacial tension, they are incompatible. Thus, the corresponding blend possessing useful properties could not be obtained by direct blending of the two polymers and there were only a few reports on the subject [14-17]. In this study, the multi-monomer grafted PP as a compatibilizer was directly added to PVC and carried on a systematic study.

Table 1 summarizes the mechanical properties of gPP/PVC blends with different proportions. In addition, the mechanical properties of PP/PVC binary blends were also given for comparison. From it we could see that, although the tensile strength of pure PVC (45.00 MPa) was higher than that of PP $(35.17 \mathrm{MPa})$, the tensile strength of $\mathrm{PP} / \mathrm{PVC}$ blends significantly reduced with the PVC content increasing. Therefore, it was impossible to obtain the good material by direct melt blending of PP and PVC. However, the tensile strengths of gPP/PVC blends were increased largely except for the weight ratio of $40 / 60$ (gPP/PVC). Namely, the multi-monomer grafted PP could improve the compatibility of PP/PVC blend and enhance the tensile strength, whereas the phase inversion played a major role at the weight ratio 40/60 of gPP/PVC. The impact strength of gPP/PVC was unchanged as that of PP/PVC with the whole study range. 
Additionally, the grafted $\mathrm{PP}$ contained $\mathrm{C}=\mathrm{O}$ groups, which should be present in the phase interfaces of blends, could form hydrogen bonding with $\mathrm{H}$ atom of $\mathrm{PVC}$, which could reduce the interfacial free energy, and decrease the interfacial tension and increase the adhesion between two different phases. On the other hand, due to the similar of dissolubility parameter $(\delta)$ about $\mathrm{PVC} \quad\left(\delta=\left(19.0_{-}\right.\right.$ $\left.22.1) \cdot 10^{-3}\left(\mathrm{Jm}^{-3}\right)^{1 / 2}\right)$ and PMMA $(\delta=$ $\left.19.0 \cdot 10^{-3}\left(\mathrm{Jm}^{-3}\right)^{1 / 2}\right)$ [11], according to the principle (the good compatibility of different polymers with the similar values), the MMA chain diffused toward PVC matrix, and the PP part in the grafted $\mathrm{PP}$ produced eutectic crystal with $\mathrm{PP}$, thus the grafted PP could take the most optimum conformations at the interface and play its compatibility role

Table 2. Effect of gPP or PP contents on the $T_{g}$ of PVC in gPP or PP/PVC blends

\begin{tabular}{|c|c|c|c|c|}
\hline $\begin{array}{c}\text { The weight ratio of PP } \\
\text { (or gPP)/PVC blends }\end{array}$ & $\mathbf{8 0 / 2 0}$ & $\mathbf{6 0 / 4 0}$ & $\mathbf{4 0 / 6 0}$ & $\mathbf{2 0 / 8 0}$ \\
\hline$T_{g(\mathrm{PVC})}$ of PP/PVC $\left[{ }^{\circ} \mathrm{C}\right]$ & 88.7 & 89.2 & 90.1 & 90.4 \\
\hline$T_{g(\mathrm{PVC})}$ of $\mathrm{gPP} / \mathrm{PVC}\left[{ }^{\circ} \mathrm{C}\right]$ & 77.9 & 79.1 & 84.3 & 86.0 \\
\hline
\end{tabular}

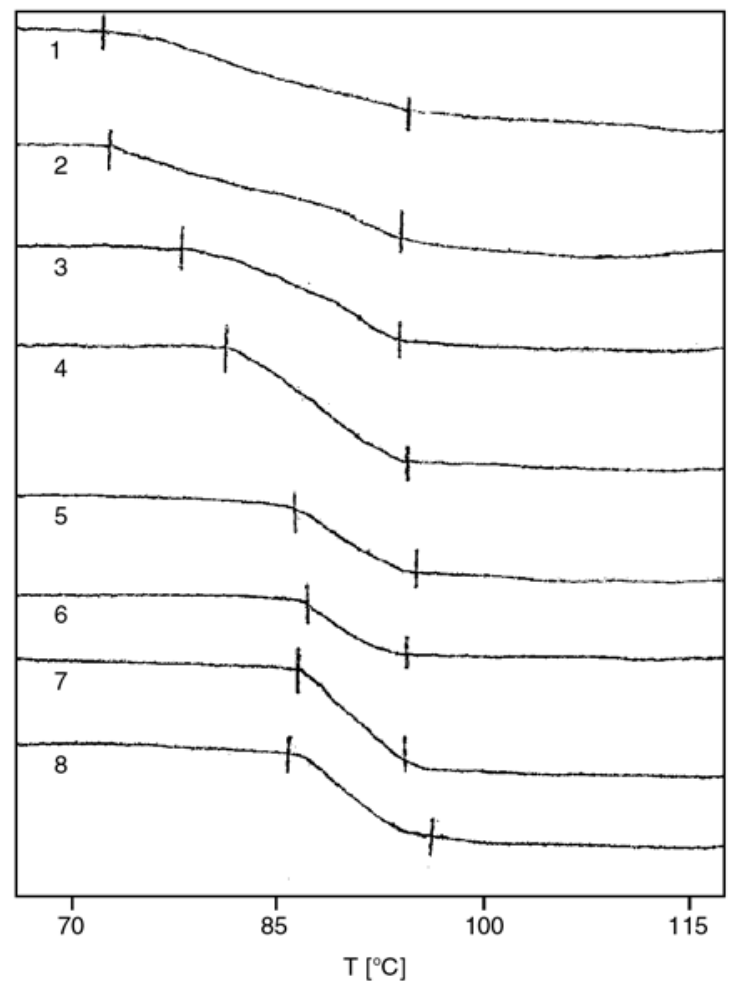

Figure 8. DSC curves for gPP/PVC and PP/PVC blends with different weight ratios. gPP/PVC:

(1) 80/20, (2) 60/40, (3) 40/60, and (4) 20/80; PP/PVC: (5) 80/20, (6) 60/40, (7) 40/60, and (8) $20 / 80$ fully [18]. Actually, the grafted polymer acted as an anchor at the interface of PP and PVC.

\subsection{DSC investigation}

Table 2 is the $T_{g}$ values of PVC portion in the two different blends, which was denoted as $T_{g(\mathrm{PVC})}$. From it we could see, due to the poor compatibility of PP and PVC, the $T_{g(\mathrm{PVC})}$ values (about $90^{\circ} \mathrm{C}$ ) were almost independent with the weight ratio of PP/PVC blends varying. However, adding the gPP to PVC, it was not difficult to find that all the $T_{g(\mathrm{PVC})}$ values reduced and tended to that of $\mathrm{PP}$ $\left(-14^{\circ} \mathrm{C}\right)$. As the weight ratio of $\mathrm{gPP} / \mathrm{PVC}$ was $80 / 20$, the $T_{g(\mathrm{PVC})}$ value decreased about $11^{\circ} \mathrm{C}$. Meanwhile, as it can be seen in Figure 8, the $T_{g(\mathrm{PVC})}$ range become wide with the gPP concentration addition. The more gPP were added, the more the $T_{g(\mathrm{PVC})}$ range broadened. All this showed that the gPP improved the compatibility of PP/PVC blends to a certain extent.

\section{Conclusions}

The multi-monomers melt grafting onto polypropylene was successfully prepared by one-step in a single-screw extruder, and characterized by means of FTIR and MFR. The effects of monomer molar ratio, monomer concentration, and initiator concentration on the grafting reaction were investigated in detail. It was shown that the addition of St to the melt-grafting system as a comonomer could significantly enhance MMA grafting degree onto PP and reduce the chain scission of $\mathrm{PP}$. The maximum MMA grafting degree was obtained when the molar ratio of [St]/[MMA] was about 1:1; and the MFR value of the gPP reached the lowest one in this case, too. As a compatibilizer, compared to pure $\mathrm{PP} / \mathrm{PVC}$ blends, the tensile strength of gPP/PVC blends was increased significantly with impact strength maintenance; expect for the phase inversion regions. Moreover, the $T_{g} \mathrm{~S}$ of DSC results also exhibited that PP-g-(St-co-MMA) improved the compatibility between PP and PVC.

\section{References}

[1] Yanjarappa M. J., Sivaram S.: Recent developments in the synthesis of functional poly(olefin)s. Progress in Polymer Science, 27, 1347-1398 (2002). 
[2] Chung T. C.: Synthesis of functional polyolefin copolymers with graft and block structures. Progress in Polymer Science, 27, 39-85 (2002).

[3] Neoh S. B., Azanam S. Hashim: Highly grafted polystyrene-modified natural rubber as toughener for polystyrene. Journal of Applied Polymer Science, 93, 1660-1665 (2004).

[4] Sun Y-J., Hu G-H., Lambla M.: Free radical grafting of methactylate onto polypropylene in a co-rotating twin screw extruder. Journal of Applied Polymer Science, 57, 1043-1057 (1995).

[5] Ciardelli F., Aglietto M., Passaglia E., Picchioni F.: Controlled functionalization of olefin/styrene copolymers through free radical processes. Polymers for Advanced Technologies, 11, 371-376 (2000).

[6] Cartier H., Hu G-H.: Styrene-assisted melt free radical grafting of glycidgl methacrylate onto polypropylene. Journal of Polymer Science, Part A: Polymer Chemistry, 36, 1053-1063 (1997).

[7] Hu G-H., Cartier H.: Styrene-assisted melt free radical grafting of glycidyl methacrylate onto an ethylene and propylene rubber. Journal of Applied Polymer Science, 71, 125-133 (1999).

[8] Cartier H., Hu G-H.: Styrene-assisted free radical grafting of glycidyl methacrylate onto polyethylene in the melt. Journal of Polymer Science Part A: Polymer Chemistry, 36, 2763-2774 (1998).

[9] Li Y., Xie X-M., Guo B-H.: Study on styrene-assisted melt free-radical grafting of maleic anhydride onto polypropylene. Polymer, 42, 3419-3425 (2001).
[10] Fu Z-S., Fan Z-Q., Zhang Y-Q., Feng L-X.: Structure and morphology of polypropylene/poly(ethylene-copropylene) in situ blends synthesized by spherical Ziegler-Natta catalyst. European Polymer Journal, 39, 795-804 (2003).

[11] Liu C., Zou H. W., Liu P. B.: Study on mechanical properties and optical transparence of PVC/SBS/MBS blend. China Plastics Industry, 35, 17-19 (2007).

[12] Chen L-F., Wong B., Baker W. E.: Melt grafting of glycidgl methacrylate onto polypropylene and reactive compatibilization of rubber. Polymer Engineer and Science, 36, 1594-1607 (1996).

[13] Xie X-M., Chen N-H., Guo B-H., Li S.: Study on multi-monomers melt-grafting onto PP in an extruder. Polymer International, 49, 1677-1683 (2000).

[14] Chung T. C., Rhubright D.: Polypropylene-graft-polycaprolactone: Synthesis and applications in polymer blends. Macromolecules, 27, 1313-1319 (1994).

[15] Kim B. J., White J. L.: Compatibilized blends of PVC/PA12 and PVC/PP containing poly(lauryl lactam-block-caprolactone). Journal of Applied Polymer Science, 91, 1983-1992 (2004).

[16] Xanthos M.: Interfacical agents for multiphase polymer systems: Recent advances. Polymer Engineer and Science, 28, 1392-1399 (1988).

[17] Byong J. K., James L. W.: Compatibilized blends of PVC/PA12 and PVC/PP containing poly(lauryl lactam-block-caprolactone). Journal of Applied Polymer Science, 91, 1983-1992 (2004).

[18] Tung T., Chen H., Zhang X., Li L., Huang B.: Molecular state of graft copolymers in the interfacial area of polyolefin and polar polymer. Polymer, 35, 4240 4242 (1994). 